\title{
Effect of Chlorella vulgaris on gut microbiota through a simulated in vitro digestion process
}

\author{
Jong Beom Jin ${ }^{1} \cdot$ Jin Wook Cha ${ }^{1} \cdot$ Il-Shik Shin ${ }^{2} \cdot$ Jin Young Jeon ${ }^{3}$. \\ Hye Suck An ${ }^{4} \cdot$ Kwang Hyun Cha ${ }^{1}$ Cheol-Ho $\operatorname{Pan}^{1}$ (D)
}

Received: 11 January 2021 / Accepted: 15 January 2021 / Published Online: 31 March 2021

(C) The Korean Society for Applied Biological Chemistry 2021

\begin{abstract}
The diet plays a fundamental role in the formation of the gut microbiota, determining the interrelationship between the gut microbiota and the host. The current study investigated the effect of Chlorella vulgaris on the gut microbiota by using simulated in vitro digestion and colonic fermentation. Bioaccessibility was measured after in vitro digestion, and SCFAs and microbial profiling were analyzed after colonic fermentation. The bioaccessibility of $C$. vulgaris was $0.24 \mathrm{~g} / \mathrm{g}$. The three major SCFAs (acetate, propionate, and butyrate) increased significantly when compared to the control group. In microbial profiling analysis, microorganisms such as Faecalibacterium, Dialister, Megasphaera, Dorea, Odoribacter, Roseburia, Bifidobacterium, Butyricmonas, and Veillonella were high in C. vulgaris group. Among them, Faecalibacterium, Dialister, Megasphaera, Roseburia, and Veillonella were thought to be closely associated with the increased level of SCFAs. Finally, it can be expected to help improve gut microbiota and health through ingestion of $C$. vulgaris. However, further studies are vital to confirm the changes in the gut microbiota in in vivo, when $C$. vulgaris is ingested.
\end{abstract}

Cheol-Ho Pan $(\bowtie)$

E-mail: panc@kist.re.kr

${ }^{1}$ Natural Product Informatics Research Center, KIST Gangneung Institute of Natural Products, Gangneung 25451, Republic of Korea

${ }^{2}$ Department of Marine Food Science and Technology, Gangneung-Wonju National University, Gangneung 25457, Republic of Korea

${ }^{3}$ Research \& Technology Division Ingredient Research Department 2 , Ingredient Business Unit, Daesang Corp, 17384, Republic of Korea

${ }^{4}$ Marine Biology Research Division, National Marine Biodiversity Institute of Korea, Seocheon 33662, Republic of Korea

This is an Open Access article distributed under the terms of the Creative Commons Attribution Non-Commercial License (http://creativecommons. org/licenses/by-nc/3.0/) which permits unrestricted non-commercial use, distribution, and reproduction in any medium, provided the original work is properly cited.
Keywords Bioaccessibility $\cdot$ Gut microbiota $\cdot$ Microbial profiling - Short chain fatty acids

\section{Introduction}

Microorganisms that live in the human gut make important contributions to human metabolism and are able to be potential substances for new therapeutic agents [1]. Gut microorganisms perform several beneficial functions in the body's metabolic, immunological, structural and neurological environment, and have a significant impact on an individual's physical and mental health [2]. One of the main features of the gut microorganisms is to ferment undigested food ingredients into short-chain fatty acids (SCFAs) such as acetate, propionate, and butyrate [3]. In particular, acetate, propionate, and butyrate are SCFAs most often discovered in feces and play a very different but important role in the human body [4].

Diet plays a fundamental role in the formation of the gut microbiota and determines the interrelationship between the gut microbiota and the host [5]. Studies of gut microbiota through the diet of animals or humans give the most reliable results, but are time consuming and expensive. In vitro studies are an alternative solution to animal or human models because they screen food ingredients in a short time [6].

Chlorella, a single-celled green alga that grows primarily in aquatic environments, provided the antioxidant activity [7]. Chlorella vulgaris is the most known Chlorella species and rich in dietary fiber, amino acids, proteins, minerals, vitamins, and unsaturated fatty acids [7]. However, how they can be changed or modulated during digestion and fermentation in human body is still not clear. Thus, the current study investigated the effect of $C$. vulgaris on the gut microbiota after applying in vitro digestion and colonic fermentation to imitate digestion. 


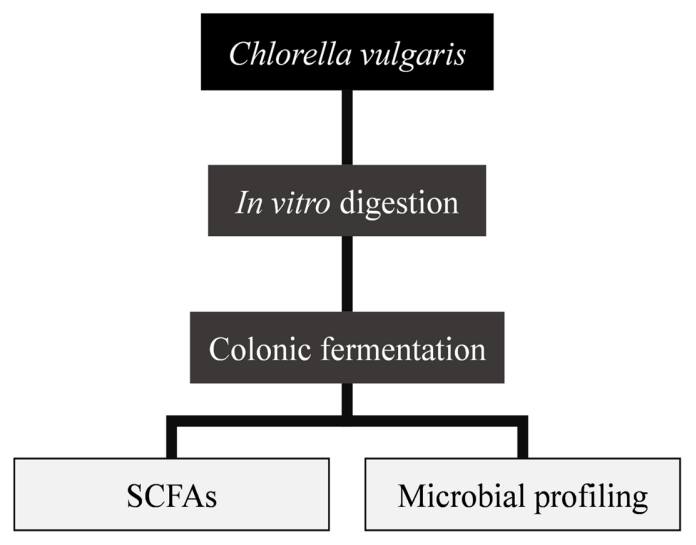

Fig. 1 Overall experimental design. The oral and gastrointestinal stages were simulated during in vitro digestion. Short chain fatty acids (SCFAs) were analyzed using gas chromatography-frame ionization detector (GCFID). Microbial profiling analysis was carried out through mi-seq

\section{Materials and Methods}

The overall experimental procedure is shown in Fig. 1.

\section{Chlorella vulgaris}

C. vulgaris was received from Daesang Corporation (Seoul, Korea). Samples were obtained in powder form and kept in a refrigerator at $4{ }^{\circ} \mathrm{C}$.

\section{In vitro digestion}

In vitro digestion was carried out using the method of PerezBurillo [8]. Three solutions (simulated saline fluid (SSF), simulated gastric fluid (SGF), and simulated intestinal fluid (SIF)) were prepared in advance by referring to Perez-Burillo's protocol. In the experiment, each $1 \mathrm{~g} \mathrm{C}$. vulgaris was transferred to a $50 \mathrm{~mL}$ conical tube, and then combined with $4 \mathrm{~mL}$ distilled water. After injecting $5 \mathrm{~mL}$ SSF solution at the oral stage, it was mixed with $\alpha$-amylase $(150 \mathrm{U} / \mathrm{mL})$ and $3 \mathrm{M} \mathrm{CaCl}_{2}(25 \mu \mathrm{L})$. Thereafter, the solution was incubated for $2 \mathrm{~min}$ in a shaking water bath (Model BS-21, JEIO TECH, Daejeon, Korea) under conditions of $37{ }^{\circ} \mathrm{C}$ and $95 \mathrm{rpm}$. In gastrointestinal stage, the cultured solution and SGF $(10 \mathrm{~mL})$ solution containing pepsin $(4000 \mathrm{U} / \mathrm{mL})$ were mixed with $4 \mathrm{~N} \mathrm{HCl}$ to lower the $\mathrm{pH}$ to 2.5 . Then, the solution was incubated in a shaking water bath under the conditions at $37^{\circ} \mathrm{C}$ and $95 \mathrm{rpm}$ for $2 \mathrm{~h}$. In small intestinal stage, SIF $(20 \mathrm{~mL})$ solution containing $20 \mathrm{mM}$ bile salt, $26.74 \mathrm{mg} / \mathrm{mL}$ pancreatin, and $1 \mathrm{~mL}$ of $0.01 \mathrm{~g} / \mathrm{mL}$ pancreatin lipase was mixed with $8 \mathrm{~N} \mathrm{NaOH}$ to make it between $\mathrm{pH} 6$ and 6.5. Then, the solution was incubated for $2 \mathrm{~h}$ in shaking water bath under conditions of $37^{\circ} \mathrm{C}$ and $95 \mathrm{rpm}$. Finally, the solution was centrifuged $\left(4{ }^{\circ} \mathrm{C}, 2000 \times g\right.$, $30 \mathrm{~min}$ ) to separate the precipitate, which was stored in $-20{ }^{\circ} \mathrm{C}$ freezer.

\section{Estimation of bioaccessibility}

Bioaccessibility was measured by investigating differences in weight changes after in vitro digestion. The sample weight was measured using an electronic balance, and after in vitro digestion, the weight was calculated by measuring the moisture through Moisture Analyzer HE78 (Mettler-Toledo, Columbus, OH, USA). Bioaccessibility was calculated through the following equation.

Bioaccessibility $(\mathrm{g} / \mathrm{g})$

$=1-\frac{\text { Sample weight after in vitro digestion }(\mathrm{g}) \times \text { Moisture content }}{\text { Sample weight before in vitro digestion }(\mathrm{g})}$

\section{Colonic fermentation}

Colonic fermentation was carried out using Long's method [9]. It was the control group that performed colonic fermentation without adding anything. Briefly, $\mathrm{NaCl}(8 \mathrm{~g} / \mathrm{L}), \mathrm{KCl}(0.2 \mathrm{~g} / \mathrm{L})$, $\mathrm{Na}_{2} \mathrm{HPO}_{4}(1.15 \mathrm{~g} / \mathrm{L}), \mathrm{KH}_{2} \mathrm{PO}_{4}(0.2 \mathrm{~g} / \mathrm{L})$, and L-cysteine $(0.5 \mathrm{~g} / \mathrm{L})$ were dissolved in distilled water and then autoclaved (phosphatebuffered saline (PBS) medium). Fecal samples were gained from three healthy donors (20-30 years old; mean body mass index 22.3) who did not take any probiotics or antibiotics for 3 months prior to the experiment. Written consent was received from each donor, and the research was approved by the Institutional Review Board (IRB No. 2015-003) of the Korea Institute of Science and Technology. Fecal samples were diluted using PBS solution to a concentration of $10 \%(\mathrm{w} / \mathrm{v})$ in an anaerobic chamber (Coy Laboratory Products Inc., Ann Arbor, MI, USA). After in vitro digestion, a portion of the precipitate $(0.5 \mathrm{~g})$ was diluted with an anaerobic PBS solution $(10 \mathrm{~mL})$. Then, the two solutions $(10 \mathrm{~mL}$ each) were mixed. Colonic fermentation of all solutions was performed at $37{ }^{\circ} \mathrm{C}$ using AnaeroPack (Mitsubishi Gas Chemical, Tokyo, Japan). All samples ( $1 \mathrm{~mL}$ ) were taken at $0,6,12$ and $24 \mathrm{~h}$ after fermentation and stored in a freezer $\left(-20^{\circ} \mathrm{C}\right)$.

\section{Profiling of short chain fatty acids (SCFAs)}

All steps from sample preparation to gas chromatography-frame ionization detector (GC-FID) analysis were carried out according to David's method [10]. First, the supernatant of the fecal mixture cultured for $0,6,12$, and $24 \mathrm{~h}$ was separated by centrifugation $\left(18,000 \times g, 4{ }^{\circ} \mathrm{C}, 10 \mathrm{~min}\right)$. Then, the supernatant $(500 \mu \mathrm{L})$ was combined with $50 \%$ internal standard (1\% 2 methylpentanoic acid), sulfuric acid $(50 \mu \mathrm{L})$, and diethyl ether $(500 \mu \mathrm{L})$. In addition, quantitative data were obtained using a $10 \mathrm{mM}$ Volatile free acid mix (Sigma-Aldrich, St. Louis, MO, USA). The mixture was moved to a vial for gas chromatography analysis. Gas chromatography equipment (300-MS, Bruker, Madison, WI, USA) was utilized for GC-FID analysis. The oven temperature was kept at $170{ }^{\circ} \mathrm{C}$ and the both injector and detector were set at $225^{\circ} \mathrm{C}$. A Nukol column (Supelco, Merck, Darmstadt, Germany; a $30 \mathrm{~m} \times 0.25 \mathrm{~mm}$ fused silica capillary column coated with a 0.25 
$\mu \mathrm{m}$ film thickness) was used for GC-FID analysis. Data were analyzed together with internal standard (1\% 2-methyl pentanoic acid) and Volatile free acid mix data to acquire quantitative data.

\section{S rRNA gene sequencing}

DNA was extracted from the precipitate acquired by centrifugation $\left(18,000 \times g, 4{ }^{\circ} \mathrm{C}, 10 \mathrm{~min}\right)$ of colonic fermented samples for $24 \mathrm{~h}$ using QIAamp DNA Stool Mini Kit (QIAGEN, Germantown, MD, USA) with bead-beating. The V3-V4 region (319F/806R) of the 16S rRNA gene was amplified by improved dual-indexing amplification [11]. Polymerase chain reaction products were purified using AMPure XT beads (Beckman, Danvers, MA, USA) and quantified using Qubit dsDNA high sensitivity reagent (Invitrogen, Carlsbad, CA, USA). Sequencing was carried out on the MiSeq platform using a paired-end $2 \times 300$-bp reagent kit (Illumina, San Diego, CA, USA). Raw reads were demultiplexed and assembled. The quality was filtered by QIIME 2 (v2018.6) using the default settings [12]. To classify the filtered reads into taxonomic groups, a Naïve Bayes classifier was trained using the
16S rRNA region (V3-V4), a primer set, read length (469 bp), and the Greengenes 97\% reference set (v13.5) [13]. This trained feature classifier was used to assign a classification to each read using the default settings of QIIME. MicrobiomeAnalyst [14] was used to analyze specific levels of microbial composition and $\beta$ diversity. $\beta$-diversity analysis method under the following conditions; Ordination method: PCoA, distance method: Jaccard Index, Taxonomic level: Feature-level, Statistical method: Permutational MANOVA (PERMANOVA). Linear discriminant analysis (LDA) Effect Size (LEfSe) analysis was performed under the following conditions for classification of human microbial community data; P-value cutoff: 0.1, FDR-adjusted, log LDA score: 2.0, number of top features: 22.

\section{Statistical analysis}

The grouped data were statistically analyzed using GraphPad Prism 9. To statistically analyze the results of SCFAs, significance was measured using the unpaired $\mathrm{t}$ test $(p<0.05)$. In addition, MicrobiomeAnalyst was used to perform microbial data processing
(A)

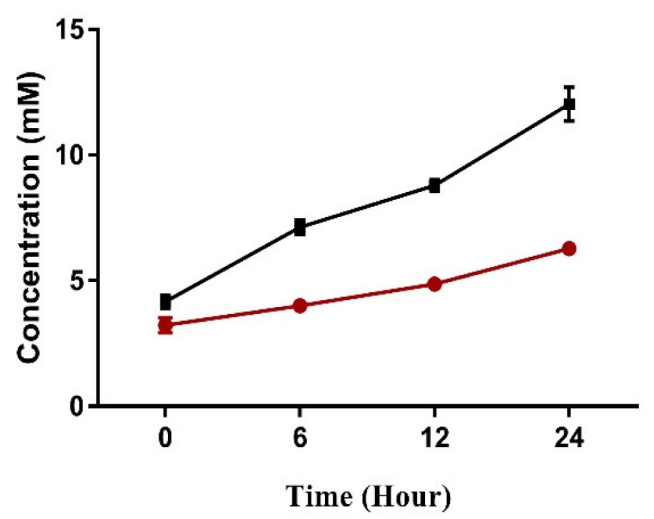

(C)

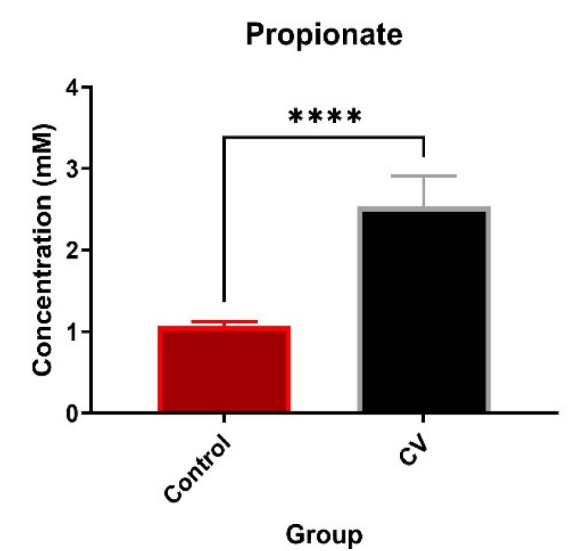

(B)

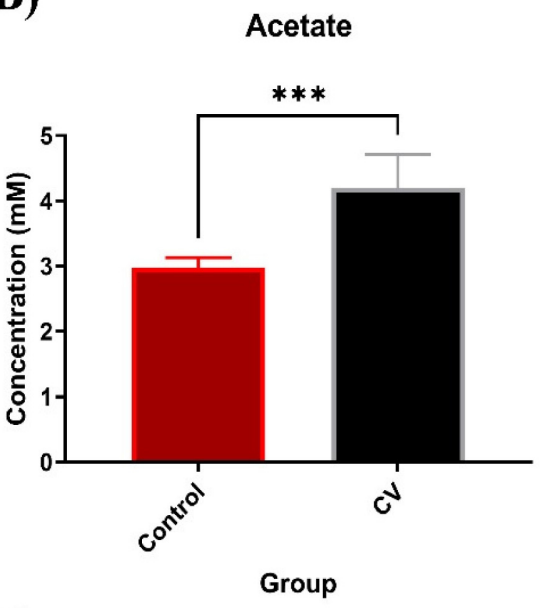

(D)

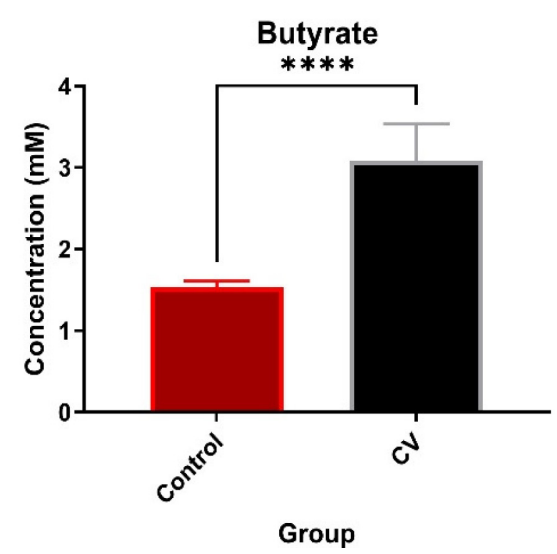

Control

Chlorella vulgaris

Fig. 2 Changes in the amount of (A) total of three major SCFAs, (B) acetate, (C) propionate, and (D) butyrate after 24 h colonic fermentation of $C$. vulgaris $(\mathrm{n}=6)$. Control: a group subjected to colonic fermentation without adding anything. $(\mathrm{CV})$ : a group subjected to colonic fermentation by adding C. vulgaris. Significance was determined using the unpaired $t$ test $\left(* * * p<0.001,{ }^{*} * * * p<0.0001\right)$ 


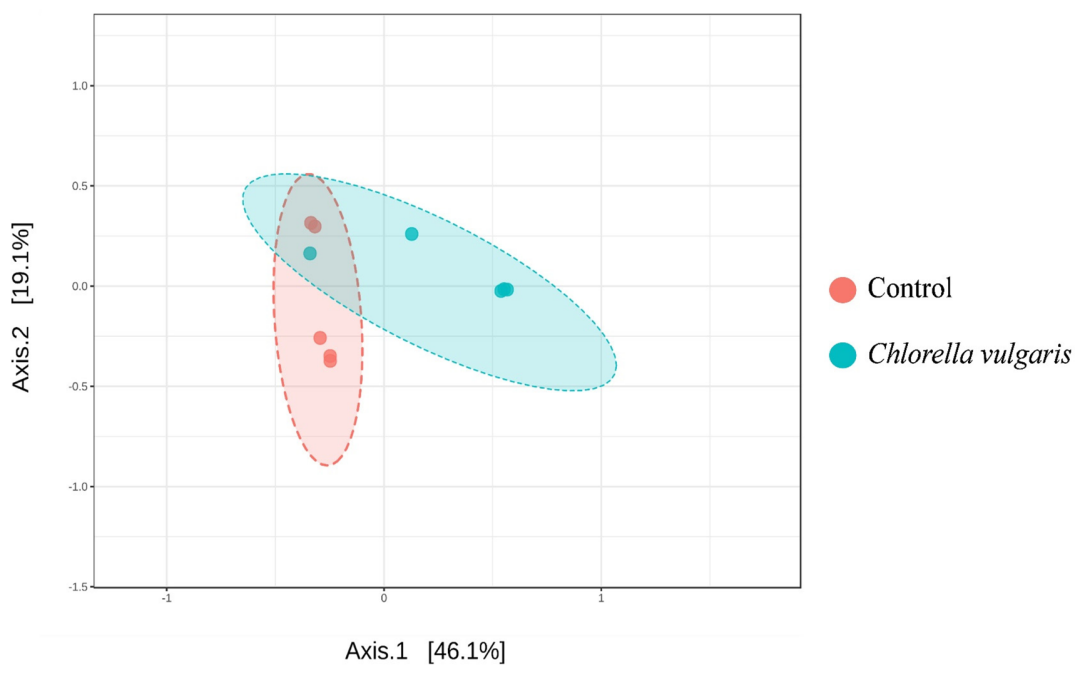

Fig. $3 \beta$-diversity analysis for two colonic fermentation groups (control and C. vulgaris). Ordination method: PCoA, Distance method: Jaccard Index, Taxonomic level: Feature-level, Statistical method: Permutational MANOVA (PERMANOVA), F-value: 3.3868; R-squared: 0.29743 ; $p<0.053$. Control: a group subjected to colonic fermentation without adding anything. Chlorella vulgaris: A group subjected to colonic fermentation by adding $C$. vulgaris.

and statistical analysis. Permutational multivariate analysis of variance (PERMANOVA) was carried out to test the difference in overall microbial composition between the control group and the C. vulgaris-treated group based on PCoA.

\section{Results and Discussion}

\section{Estimation of bioaccessibility}

The bioaccessibility $(\mathrm{g} / \mathrm{g})$ of $C$. vulgaris, relative amount measured before entering colon stage after in vitro digestion, was $0.24 \mathrm{~g} / \mathrm{g}$. It implies that $0.76 \mathrm{~g}$ of residual component could reach the large intestine, possible to interact with gut microbes when $1 \mathrm{~g}$ of $C$. vulgaris is consumed.

\section{Profiling of SCFAs}

After colonic fermentation, SCFAs which are major products of gut microorganisms were analyzed. As the fermentation time passed, the difference in concentration of three major SCFAs between the control and $C$. vulgaris group increased more and more (Fig. 2A). After $24 \mathrm{~h}$ colonic fermentation, the difference in concentration of acetate, propionate, and butyrate was also significant $(p<0.05)$ (Fig. 2B-D); acetate: control $(2.979 \mathrm{mM} \pm$ $0.063, \mathrm{n}=6$ ), C. vulgaris (4.199 $\mathrm{mM} \pm 0.210, \mathrm{n}=6$ ), $p<0.001$; propionate: control $(1.069 \mathrm{mM} \pm 0.023, \mathrm{n}=6), C$. vulgaris $(2.536$ $\mathrm{mM} \pm 0.152, \mathrm{n}=6), \quad p<0.0001$; butyrate: control $(1.535 \mathrm{mM} \pm$ $0.030, \mathrm{n}=6), C$. vulgaris $(3.085 \mathrm{mM} \pm 0.184, \mathrm{n}=6), p<0.0001$. It was predicted that there is a clear difference in the microbial community because the difference in SCFAs is significant.

\section{Measurement of $\boldsymbol{\beta}$-diversity}

Among the microbial profiling analysis results, $\beta$-diversity was analyzed to decide whether or not there is a difference between the microbial communities of two groups (control and C. vulgaris) (Fig. 3). As a result of the analysis, it was confirmed that there was a difference between the microbial communities in two groups.

\section{LEfSe analysis}

Linear discriminant analysis (LDA) Effect Size (LEfSe) analysis was performed to find the microorganisms that influenced the difference between the microbial communities for the two groups (Fig. 4). LefSe analysis was conducted under the following conditions; P-value cutoff: 0.1, FDR-adjusted, log LDA score: 2.0, number of top features: 22. In LefSe analysis, all results were analyzed at genus level. 9 microorganisms such as Faecalibacterium, Dialister, Megasphaera, Dorea, Odoribacter, Roseburia, Bifidobacterium, Butyricmonas, and Veillonella were high in C. vulgaris group. 13 microorganisms such as Lactococcus, cc_115, Streptococcus, Mucispirillum, Anaerotruncus, Blautia, Dehalobacterium, Coprobacillus, Enterococcus, Coprobacillus, Adlercreutzia, Allobaculum, and Prevotella were high in the control group. Microorganisms which are high in $C$. vulgaris group were predicted to have an effect on the increase in the concentration of SCFAs.

\section{Analysis of discriminative microorganisms from $C$. vulgaris group}

The association between the microbes and SCFAs production was examined. Five microorganisms (Faecalibacterium, Dialister, Megasphaera, Roseburia, and Veillonella) were selected by comparing the results obtained from LEfSe analysis and the result by Reichardt et al. [15] in which the microorganisms related to propionate or butyrate production are summarized. After that, the relative abundance of those microbes was shown in Fig. 5 by 


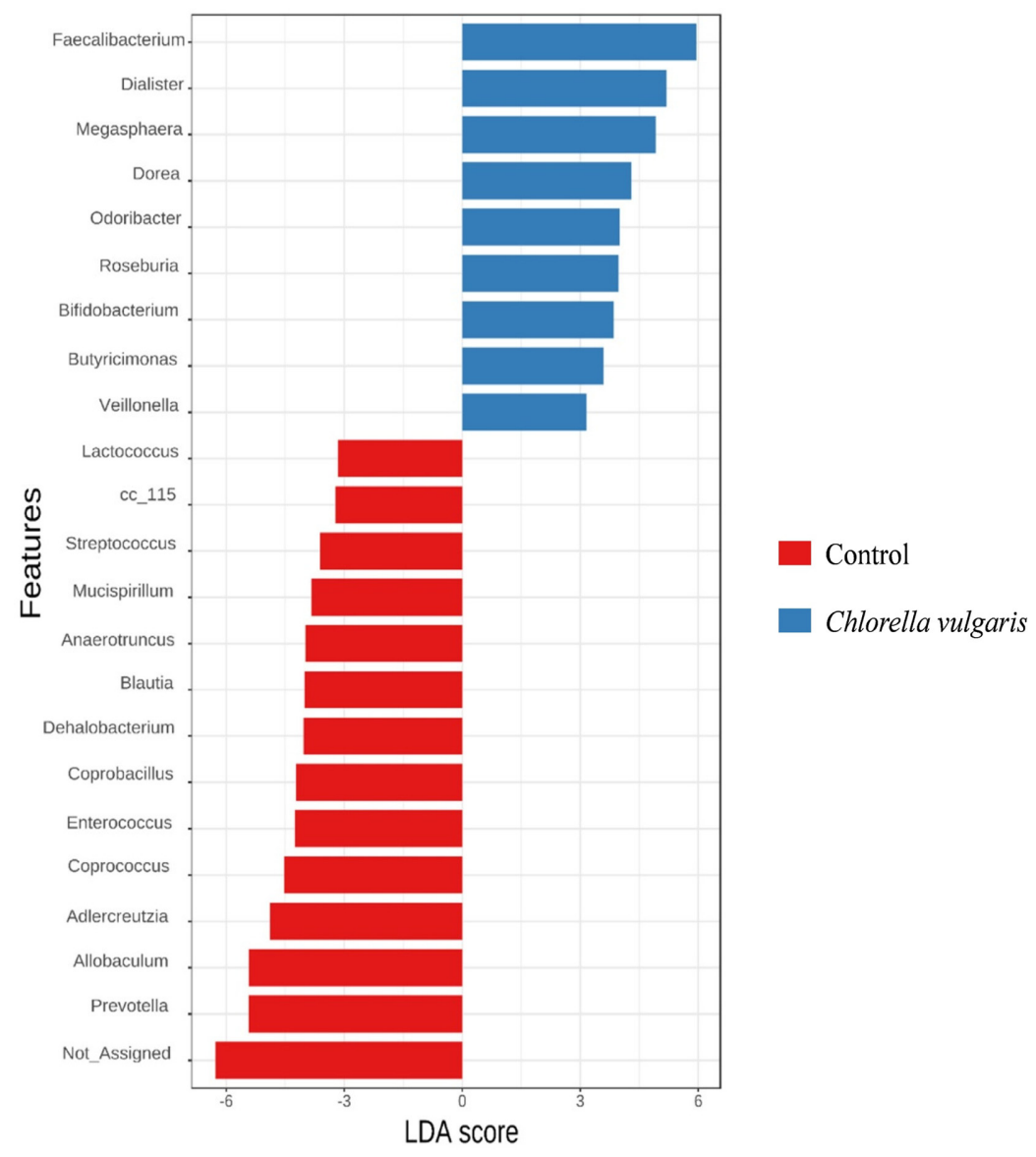

Fig. 4 LEfSe analysis for two colonic fermentation groups. $p$-Value cutoff: 0.1 , FDR-adjusted, log LDA score: 2.0 , number of top features: 22. Control: A group subjected to colonic fermentation without adding anything. Chlorella vulgaris: A group subjected to colonic fermentation by adding C. vulgaris.

comparing the control group with the $C$. vulgaris group. Microorganisms associated with propionate production are Dialister, Veillonella, and Megasphaera. Propionate is primarily metabolized in the liver and plays a role in reducing the concentration of blood sugar and serum cholesterol [16]. In addition, propionate is vital in regulating endocrine production of adipose tissue and preventing the metabolic diseases such as obesity [17]. Microorganisms associated with butyrate production are Faecalibacterium and Roseburia. Butyrate is a main energy source for colon cells and has a direct effect on cell growth and differentiation [18]. In addition, butyrate is known to be effective in preventing colon cancer [19].

Probiotics are defined as living microorganisms that, when administered in appropriate amounts, provide a health benefit to the host [20]. In general, Lactobacillus and Bifidobacterium have been used as probiotics [21]. Bifidobacteria are known to have beneficial influences on humans through protection against pathogens, synthesis of important vitamins, inhibition of potential toxicity, digestion of plant oligosaccharides/polysaccharides and carcinogenic metabolites, and stimulation of host immune response
[22]. As a result of the LEfSe analysis in Fig. 4 and 5, Bifidobacterium was higher in the $C$. vulgaris group compared to the control group. Therefore, it was expected that the ingestion of C. vulgaris helped improve gut microbiota community and human health.

This study suggests that taking $C$. vulgaris may improve the intestinal microbial flora. The bioaccessibility of $C$. vulgaris was obtained through in vitro digestion, and the amount of $C$. vulgaris in the colon was predicted. After colonic fermentation, SCFAs analysis confirmed an increase in high SCFAs in the group to which Chlorella vulgaris was added. Microbial profiling analysis using MiSeq confirmed that the gut microbiota was changed by addition of $C$. vulgaris. Based on our results, ingestion of $C$. vulgaris can help people's health through improving the intestinal microbial flora. However, further studies are vital to confirm the changes in the gut microbiota in in vivo, when $C$. vulgaris is ingested.

Acknowledgment This work was supported by the Ministry of Oceans and Fisheries, Korea [grant number 20170488]. 
(A)

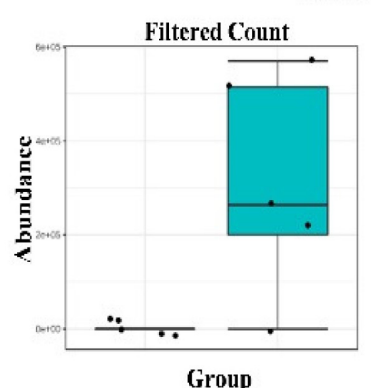

(C)

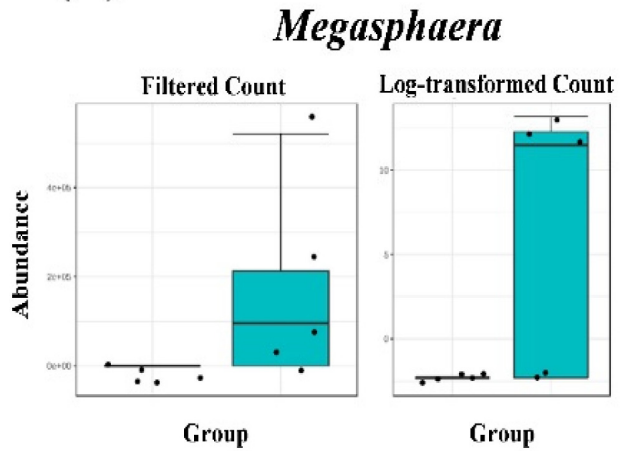

(E)

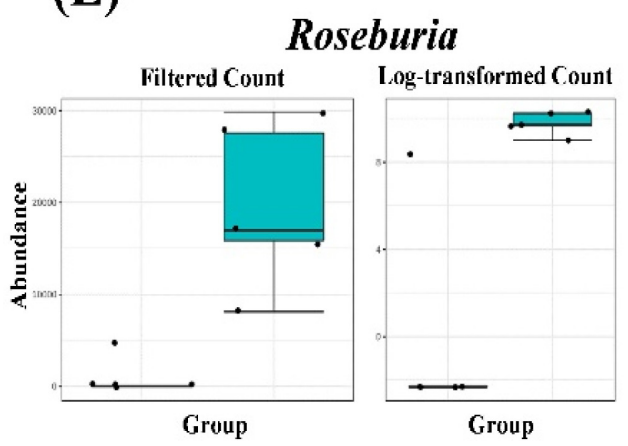

(B)

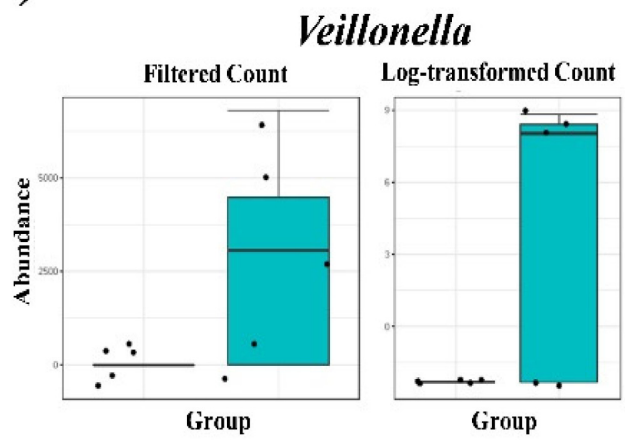

(D)

Faecalibacterium

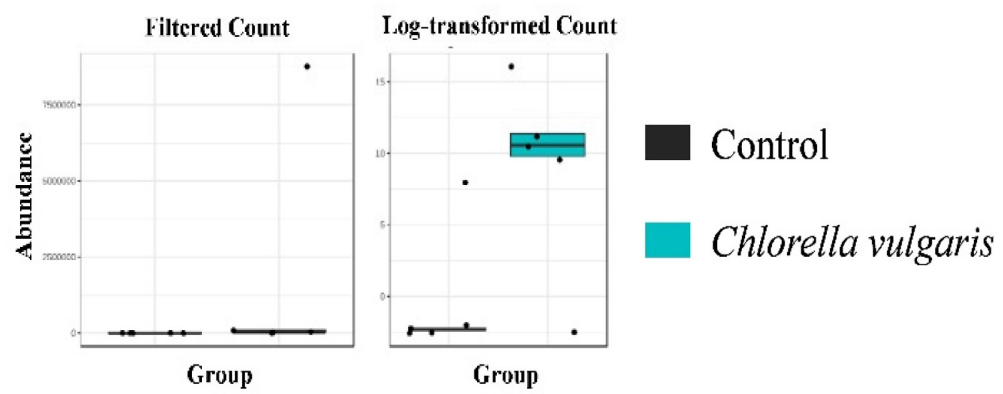

(F)

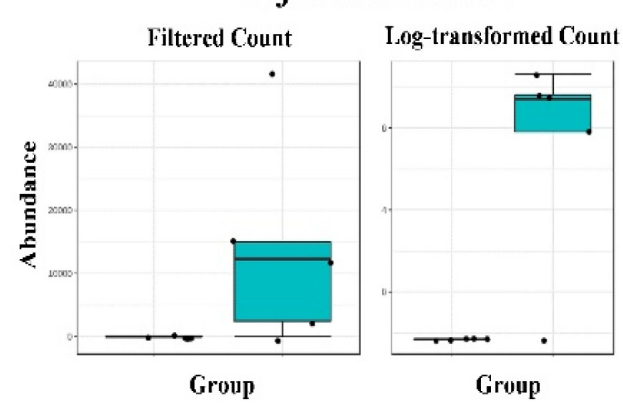

Fig. 5 The relative abundance of six discriminative taxa, Dialister, Veillonella, Megasphaera, Faecalibacterium, Roseburia, and Bifidobacterium.

\section{References}

1. Cani PD (2018) Human gut microbiome: hopes, threats and promises Gut 67: 1716-1725

2. Adak A, Khan MR (2019) An insight into gut microbiota and its functionalities. Cell Mol Life Sci 76: 473-493

3. van der Beek CM, Dejong CHC, Troost FJ, Masclee AAM, Lenaerts K (2017) Role of short-chain fatty acids in colonic inflammation, carcinogenesis, and mucosal protection and healing. Nutr Rev 75: 286305

4. Rowland I, Gibson G, Heinken A, Scott K, Swann J, Thiele I, Tuohy K (2017) Gut microbiota functions: metabolism of nutrients and other food components. Eur J Nutr 57: 1-24
5. Ercolini D, Fogliano V (2018) Food design to feed the human gut microbiota. J Agr Food Chem 66: 3754-3758

6. Lyu Y, Bi J, Chen Q, Wu X, Qiao Y, Hou H, Zhang X (2021) Bioaccessibility of carotenoids and antioxidant capacity of seed-used pumpkin byproducts powders as affected by particle size and corn oil during in vitro digestion process. Food Chem 343: 128541

7. Panahi Y, Darvishi B, Jowzi N, Beiraghdar F, Sahebkar A (2016) Chlorella vulgaris: a multifunctional dietary supplement with diverse medicinal properties. Curr Pharm Des 22: 164-173

8. Pérez-Burillo S, Rufián-Henares JA, Pastoriza S (2018) Towards an improved global antioxidant response method (GAR+): Physiologicalresembling in vitro digestion-fermentation method. Food Chem 239: 1253-1262

9. Long W, Xue Z, Zhang Q, Feng Z, Bridgewater L, Wang L, Zhao L, 
Pang X (2015) Differential responses of gut microbiota to the same prebiotic formula in oligotrophic and eutrophic batch fermentation systems. Sci Rep 5: 13469

10. David LA, Maurice CF, Carmody RN, Gootenberg DB, Button JE Wolfe BE, Ling AV, Devlin AS, Varma Y, Fischbach MA, Biddinger SB, Dutton RJ, Turnbaugh PJ (2014) Diet rapidly and reproducibly alters the human gut microbiome. Nature 505: 559-563

11. Fadrosh DW, Ma B, Gajer P, Sengamalay N, Ott S, Brotman RM, Ravel J (2014) An improved dual-indexing approach for multiplexed $16 \mathrm{~S}$ rRNA gene sequencing on the Illumina MiSeq platform. Microbiome 2: 6

12. Kuczynski J, Stombaugh J, Walters WA, Gonzalez A, Caporaso G Knight R (2011) Using QIIME to analyze 16S rRNA gene sequences from microbial communities. Curr Protoc Bioinform. 36: 10.7.1-10.7.20

13. DeSantis TZ, Hugenholtz P, Larsen N, Rojas M, Brodie EL, Keller K, Huber T, Dalevi D, Hu P, Andersen GL (2006) Greengenes, a chimerachecked 16S rRNA gene database and workbench compatible with ARB. Appl Environ Microbiol 72: 5069-5072

14. Dhariwal A, Chong J, Habib S, King IL, Agellon LB, Xia J (2017) MicrobiomeAnalyst: a web-based tool for comprehensive statistical, visual and meta-analysis of microbiome data. Nucleic Acids Res 45: W180-W188

15. Reichardt N, Duncan SH, Young P, Belenguer A, Leitch CM, Scott KP,
Flint HJ, Louis P (2014) Phylogenetic distribution of three pathways for propionate production within the human gut microbiota. ISME $\mathrm{J} 8$ : 1323-1335

16. Ramakrishna BS (2013) Role of the gut microbiota in human nutrition and metabolism. J Gastroenterol Hepatol 28: 9-17

17. Fava F, Rizzetto L, Tuohy KM (2019) Gut microbiota and health: connecting actors across the metabolic system. Proc Nutr Soc 78: 177188

18. Fu X, Liu Z, Zhu C, Mou H, Kong Q (2019) Nondigestible carbohydrates, butyrate, and butyrate-producing bacteria. Crit Rev Food Sci Nutr 59: S130-S152

19. McNabney SM, Henagan TM (2017) Short chain fatty acids in the colon and peripheral tissues: a focus on butyrate, colon cancer, obesity and insulin resistance. Nutrients 9: 1348

20. Sanders ME, Merenstein DJ, Reid G, Gibson GR, Rastall RA (2019) Probiotics and prebiotics in intestinal health and disease: from biology to the clinic. Nat Rev Gastroenterol Hepatol 16: 605-616

21. Sarao LK, Arora M (2017) Probiotics, prebiotics, and microencapsulation: a review. Crit Rev Food Sci Nutr 57: 344-371

22. Ngo N, Choucair K, Creeden J, Qaqish H, Bhavsar K, Murphy C, Lian K, Albrethsen MT, Stanbery L, Phinney RC, Brunicardi FC, Dworkin L, Nemunaitis J (2019) Bifidobacterium spp: the promising Trojan Horse in the era of precision oncology. Future Oncol 15: 3861-3876 